\title{
Stability Conditions of Fuzzy Filter Type III
}

\author{
José de Jesús Medel Juárez , Juan Carlos García Infante², Juan Carlos Sánchez García² \\ ${ }^{1}$ Centre of Computing Research, National Polytechnic Institute, Vallejo, México; ${ }^{2}$ Professional School of Mechanical and Electrical \\ Engineering, National Polytechnic Institute, Coyoacan, México. \\ Email: jjmedelj@yahoo.com.mx, jcnet21@yahoo.com, jcsanchezgarcia@gmail.com
}

Received October $12^{\text {th }}, 2010$; revised February $10^{\text {th }}$, 2011; accepted February $17^{\text {th }}, 2011$.

\begin{abstract}
A digital fuzzy logic filter of type III interacts with a real model signal reference to obtain the best answer in the sense of minimum mean square error of the output. The key part of the filter is a fuzzy mechanism that adaptively selects and emits answer according to the changes of the external reference signal. Based on input signal level, this fuzzy filter selects the best parameter values from a set of membership in the knowledge base (KB), and the filter weights are updated according to the reference signal in a natural form. With this fuzzy structure the filter reduces error. The simulation result shows the stability of the filter. The states of the filtering process require that all of its answers are bounded by the error criteria probabilistically.
\end{abstract}

Keywords: Digital Filtering, Fuzzy Systems, Estimation, Stability

\section{Introduction}

One of the best tools used to approximate an external process, is a recursive digital filter that adjusts dynamically its parameters and weights to give the best answers with respect to the mean square error criterion [1]. A digital filter is a logic algorithm programmed into computer software or electronic to eliminate the noise of a system, taking specific data, identifying system or predicting a system $[1,2]$.

The main problem in conventional filtering operation is that it can't classify and infer its operation levels with respect to a changing reference system; interpreting its external environment changes in order to select its answers with the smallest error and considering different operation levels in dynamical sense, following the natural evolution of the reference system that interacts with the filter. In addition, a conventional filter has not a linguistic description to display its answer conditions in human readable formats, which have problems to develop capacities with high dynamical changing processes [3].

The systems related to artificial intelligent mechanisms should use fuzzy tools (as fuzzy neural net and evolutive systems) to get its own perception giving the best decision answers, using this to solve complex problems, actualizing and adapting its perceptions and answers in accordance with a reference dynamical system [4].
The fuzzy intelligent tool as learning techniques in a digital filtering is an option to obtain different decision answer levels to get the optimal answers, having an interaction with an external reference dynamical system, adapting its answers to the possible changes by selecting the best values to get the necessary convergence conditions, which should have the best operation each time [5].

The main goal of this kind of filter is to have different level answers that describe the reference system operation as natural process, using a rule set. This requires a feedback law in order to follow the basic properties of a desired input signal, adjusting its parameters to give a correct solution dynamically to minimize the error criterion response and updates the filter parameters [6].

In the fuzzy filter properties, there is a problem trying to describe the stability conditions of a fuzzy system.But using the Kharitonov [7] theory with the Routh-Hurwitz criterion in order to get the polynomials stability, it will be easier and it could be integrated into the fuzzy stage to demonstrate a system correct operation.Previous work has shown into the simulation description of the filter stability [8,9].

With this perspective, the paper integrates the FDF (Fuzzy Digital Filter) concept, using statistical methods to characterize theKalman filter internal structure to give answers with respect to the operation levels in a natural way making a specific decision to follow the natural reference model using fuzzy logic type III [10]. The paper shows the stability analysis for the fuzzy filter structure 
to establish the bounds of the filter parameters using the Kharitonov theory with the Ruth-Hurwitz criterion.

\section{Fuzzy Intelligence}

The fuzzy systems obtain their basic ideas since the first advances of fuzzy logic by Zadeh since 1965, with respect to the fuzzy sets; Fuzzy systems have an important goal the use of approximation reasoning, establishing a relation using the fuzzy mechanism interacting with the external process. According with this a fuzzy system has been applied since fifteen years ago to develop new intelligent technologies that consider a stage that interpret different operational levels [11].

A fuzzy system has stable operations delimiting all its variables and answers into the knowledge base (KB) by the process conditions; a fuzzy system has a memory of all the answer levels given by the process. The answer level classification is using a set of membership functions; each one has a specific value in accordance with the operation level interpretation at the input. Using fuzzy rules the logic connector if, interprets the input, the fuzzy mechanism deduce the output value and the logic connector then, selects the correct membership function into the KB to updates a process [12].

The fuzzy system first interprets its environment changes at its input to get a specific operation level of the reference system, having a characterization of the input signal $y(k)$ (desired signal) into levels. Then, the fuzzy stage choose the corresponding if-then rule to get the membership function value $\hat{a}(k)$ in order to select the best parameter into the knowledge base, updating the process to a new required condition [13].

The fuzzy mechanism shown in Figure 1 describes the reasoning of an external real process operation (reference model) having a set of answerslimitedinto levels. Dynamically the fuzzy stage interprets its input data describing the external process into different levels. The fuzzy stage has previously the set of all the possible values that can get from its input. Then having this membership grade to deduce the answer value to be selected, automatically makes a selection of the membership value into the knowledge base (this has all possible answers values that it can give), and with this new parameter value updates the process to a new required condition [14]. The characterization of a fuzzy system into levels could describe different kinds of process states (as low, medium or high) in accordance with the variable (temperature,

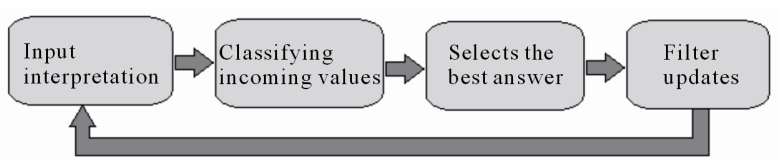

Figure 1. The fuzzy process. velocity or pressure). The fuzzy mechanism dynamically gets a corresponding answer to update a process respect to the input changes. Using the fuzzy rules in order to obtain the input level and get the corresponding answer value $\hat{y}(k)$. This process considers stable conditions because the characterization of a system uses as limit the frequency distribution probabilistically using a set of membership grades (with triangle or Gaussian functions) to obtain the operation levels [15].

The membership function in a fuzzy system is a value to choose with the minimum error using the actual input level from the reference process to update the process to a new condition. The operation levels are previously into the knowledge base of the fuzzy system as in artificial intelligence using supervised learning [16]. The goal of a this kind of systems is to classify an external reference process into grades and get the best corresponding updating answer dynamically with the best signal approximation and minimizing the errors [17]. The description of the signal into a fuzzy structure is using ranks limited by the reference system in order to characterize the signal levels as:

First using the logic connector IF to get the membership grade of the input $y(k)$, second using the logic connector THEN to get the corresponding parameter value $\hat{a}(k)$, third the fuzzy mechanism updates the process to a new condition, and gets the output value described as $\hat{y}(k)$ with the best approximation.

\section{Fuzzy Digital Filtering}

The fuzzy filter is an intelligent filter that has two stages basically; the conventional adaptive filter structure considering all its conditions, and the fuzzy stage integrated to the filter structure in order to characterize its operation to get a best signal approximation by levels. This stages integration works together minimizing the error criterion difference having a natural signal description in accordance with the reference model changes.

On the other hand, a digital filter structure without this fuzzy stage cannot have a characterization of an external process to have a normal operation without get an answer into levels, making difficult to select the best signal approximation with the minimum error. Having this is difficult to interpret the input signal of the external process and to get the best parameter value in order to update the process with the nearest value to have a better natural signal description [18].

According with this a fuzzy digital filter is an adaptive filter adding a fuzzy stage that classifies the input signal $y(k)$, into fuzzy grades to select the best filter parameter value $\hat{a}(k)$ from the knowledge base in order to update the filter weights dynamically trough time to get the best signal approximation respect to the operation 
level. A fuzzy filter classifiessearches and associates information giving the corresponding answer value according to the desired signal from the reference process at its input. This classifies a reference process in order to get a dynamical filter answers. The goal is to give a desired operation condition each time with stable operation because this filter uses as limit answer value the mean square error criterion (1) as adaptive filtering, but the fuzzy stage minimize the error difference and gets the best signal approximation. The membership functions (parameters value set) are limited into the knowledge base [19].

$$
J(k):=E\left\{\left(e(k) e(k)^{T}\right)\right\} \subseteq \Re^{2}
$$

The criterion $J(k)$ to reduce the filter error describes the mean square of the error between the desired signal $y(k)$ and the filter output $y(k)$, allows finding the corresponding membership function that is the best signal approximation to minimize the filter criterion [20]. The Fuzzy filter with adaptive properties has an iterative searching methodology using the backpropagation $(B P)$ learning algorithm, which updates its parameters per iteration dynamically by degrees classifying its membership functions into the knowledge base in accordance to the difference between the desired response $y(k)$ and the actual filter output $\hat{y}(k)$ described as the error $e(k)$. The Figure 2 shows the fuzzy filter structure:The FDF interacts with an external reference model (real process) changes and the filter will selects the best answer to approximate the signal with minimum error. Then, the fuzzy filter has next stages [21]: The fuzzy filter initially has the classification of the reference process model conditions as operation levels; having the knowledge of all the possible levels.

- It interprets the desired signal $y(k)$ operation levels with the error value $e(k)$ in probabilistic form using the inference mechanism (with the logic connector if).

- It selects a corresponding membership function $\hat{a}(k)$ (parameter value) from the knowledge base (using the logic connector then according to the error level $e(k)$.

- The parameter value $\hat{a}(k)$ selected, updates the filter operation, adapting automatically its weigh values to give a correct answer $\hat{y}(k)$, minimizing the least mean square criterion, with the best approximation value of the desired signal $y(k)$.

- The filter emits the best answer $\hat{y}(k)$, describing its responses as linguistic operational levels and continually repeats this cycle.

This computational model described as intelligent process may interacts with natural processes as biologic signals, using processing elements making connections to

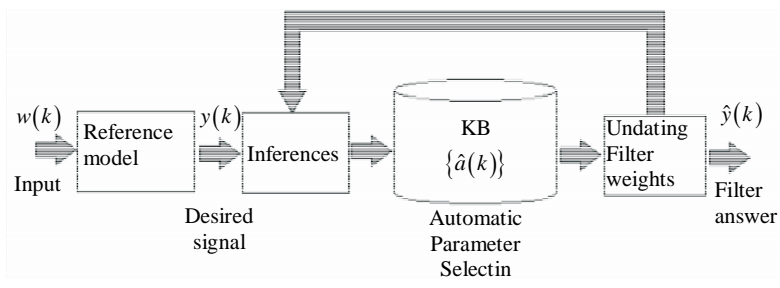

Figure 2. Fuzzy filter description.

constitute an automatic updating selection to follow the best condition of a process.

The control area $T_{N}$ is a set of answers that the filter has with respect to all set of desired signals that the reference system emit; the Control Area (2) is into membership intervals inside the knowledge base; in accordance to a filtering criterion (mean square error). The set of membership function (parameters value) represents all the correct responses into the Knowledge Base (KB), according to an objective law, predefined by this natural reference process, the filter inference chooses the best correct response from the knowledge base to each reference model change [22].

$$
T_{N}=\{(y(k), \hat{y}(k))\} \subseteq \Re^{2}
$$

The desired signal $y(k)$ at the filter input is changing its operation conditions continuously, described in levels. The filter interprets the corresponding level interval and selects the best parameter value $\hat{a}(k)$ (membership function) from the knowledge base. Updating the filter operation to give a correct response $\hat{y}(k)$, the goal of the filtering process as predictor is to follow the desired signal $y(k)$ to describe the reference process into operation grades, and then the output filter $\hat{y}(k)$ will be approximately the same signal.

This filtering process determines the best value to update the filter conditions and give a response with the smallest error.

\section{Stability Properties}

The fuzzy filtering interacting with a real process has all its answers bounded in order to give a correct parameter selection [23]. But the main problem to solve is the description of the stability analysis for a filter operation with fuzzy properties having a description of its answers into intervals. In 1978 Kharitonov made a theory for robust systems using four polynomials [7].

In accordance with the four polynomials it is possible to have a fuzzy stability description (into levels or ranks) using the Routh-Hurwitz criteria for lineal systems as fuzzy filters.

If a system has the following form:

$$
G(z)=Y(z)(\tilde{W}(z))^{-1}
$$


where the output equation described as:

$$
Y(z):=\sum_{i=1, m} b_{i} z^{-i}
$$

and its characteristic equation described as:

$$
\tilde{W}(z)=\sum_{j=1, n} a_{j} z^{-j}
$$

with $m, n \in N$

Getting from each polynomial the parameter and establishing the uncertainty values as fuzzy systems bounded into intervals. The parameters are:

$$
a_{n} \subseteq\left[\underline{a}_{n}, \bar{a}_{n},\right], \underline{\lim } a_{n}=\underline{a}_{n}, \overline{\lim } a_{n}=\bar{a}_{n}, n \in N
$$

From the polynomial described above in Equation (5), in agreement to fuzzy logic parameters and the Kharitonov theory, we get two polynomials limits:

$$
\tilde{W}(z)=\tilde{W}_{\text {Pair }}(z)+\tilde{W}_{\text {odd }}(z)
$$

Separating the pair and odd form of Equation (5) we get the next polynomials:

$$
\begin{aligned}
& \tilde{W}(z)_{\text {pair }}=a_{0}+a_{2} z^{-2}+a_{4} z^{-4}+\cdots \\
& \tilde{W}(z)_{\text {odd }}=a_{1} z^{-1}+a_{3} z^{-3}+a_{5} z^{-5}+\cdots
\end{aligned}
$$

where $\underline{a}_{n}$ and $\bar{a}_{n}$ are the limit intervals of each membership function. The nominal value of each interval $\left|\underline{a}_{n}, \bar{a}_{n}\right|$ (that is the maximum value of the membership function) is the parameter $a_{n}$. Having the characteristic equation description in robust sense as:

$$
\begin{aligned}
\tilde{W}(z) & =\left[\underline{a_{n}}, \overline{a_{n}}\right] z^{-n}+\left[\underline{a_{n-1}}, \overline{a_{n-1}}\right] z^{-(n-1)} \\
& +\left[\underline{a_{n-2}}, \overline{a_{n-2}}\right] z^{-(n-2)}+\cdots+\left[\underline{a_{0}}, \overline{a_{0}}\right]
\end{aligned}
$$

Now the composition of the Kharitonov's polynomials describing the fuzzy filter stability is:

$$
\begin{aligned}
\tilde{W}_{1,3}(z)= & \tilde{W}(z)_{1}+\tilde{W}(z)_{3}=\underline{a_{0}}+\underline{a_{1}} z^{-1}+\overline{a_{2}} z^{-2} \\
& +\overline{a_{3}} z^{-3}+\underline{a_{4} z^{-4}}+\underline{a_{5}} z^{-5}+\cdots \tilde{W}_{1,4}(z) \\
= & \tilde{W}(z)_{1}+\tilde{W}(z)_{4}=\underline{a_{0}}+\overline{a_{1}} z^{-1}+\overline{a_{2}} z^{-2} \\
& +\underline{a_{3}} z^{-3}+\underline{a_{4}} z^{-4}+\overline{a_{5}} z^{-5}+\cdots \tilde{W}_{2,3}(z) \\
= & \tilde{W}(z)_{2}+\tilde{W}(z)_{3}=\overline{a_{0}}+\underline{a_{1}} z^{-1}+\underline{a_{2}} z^{-2} \\
& +\overline{a_{3}} z^{-3}+\overline{a_{4}} z^{-4}+\underline{a_{5}} z^{-5}+\cdots \tilde{W}_{2,4}(z) \\
= & \tilde{W}(z)_{2}+\tilde{W}(z)_{4}=\overline{a_{0}}+\overline{a_{1}} z^{-1}+\underline{a_{2}} z^{-2} \\
& +\underline{a_{3}} z^{-3}+\overline{a_{4}} z^{-4}+\bar{a}_{5} z^{-5}+\cdots
\end{aligned}
$$

If all of the polynomials complain the Ruth-Hurwitz criteria, the filtering process has stability properties.

\section{Simulation}

For the simulation of the fuzzy filter, we integrate the
Kalman filter structure with the fuzzy stage characterizethe filter operation, showing graphically the fuzzy filter operation. The Kalman filter in this case uses the identification configuration, having a dynamical parameter selection to approximate the desired signal optimally. The simulation run 1000 iterations and used the set of membership functions deduce all the filter changes limited by the Error Criterion (1).The reference model into the simulation is an Auto Regressive Mobile Average (ARMA) model, which interacts with the fuzzy filter in order to get the best answers. This has a description in discrete states space, expressed by the fifth order difference as:

$$
x(k+1)=\sum_{i=0}^{5} a(k-i) x(k-i)+w(k)
$$

The fifth order corresponds to $k=5$, and its output has the next description:

$$
y(k)=x(k)+v(k), x(k), w(k), v(k) \in \mathfrak{R}
$$

where: $x(k-i)$ is the reference model internal states sequence, $a(k-i)$ the parameters sequence, $w(k)$ the reference model noise, $y(k)$ the desired signal from the reference model to the filter input, and $v(k)$ the output vector noises.

Considering the output recursive form with respect to Equations (11) and (12):

$$
y(k)=\overline{a r}(k)+\tilde{w}(k)
$$

where: $\quad \tilde{w}(k)=f(\{v(k-i)\}, \quad w(k-1), \quad v(k)) \subseteq$ $N\left(\mu_{\tilde{w}}, \sigma_{\tilde{w}}^{2}<\infty\right), \quad y(k), \quad \tilde{w}(k) \in \mathfrak{R}$, $\bar{a}:=[a(k-1)|\cdots| a(0)] \subseteq \mathfrak{R}^{[k \times 1]}$

$r(k):=[y(k-1)|\cdots| y(0)]^{T} \subseteq \mathfrak{R}^{[1 \times k]}$ and Equation with upper $(U)$ and lower $(L)$ boundaries.

$$
\begin{aligned}
& \bar{y}(k)=\bar{a}_{U} \bar{r}(k)+\tilde{w}(k) \\
& \underline{y}(k)=\bar{a}_{L} \bar{r}(k)+\tilde{w}(k),
\end{aligned}
$$

Respectively, where $\bar{a}_{U}$ and $\bar{a}_{L}$ are the boundaries corresponding to each membership function. The nominal value set of this interval $\left[\bar{a}_{L}, \bar{a}_{U}\right]$ (that is the maximum value of the membership function allowed) is the parameters vector $\bar{a}$.

Now considering in Equation (13) the Z-transform, the characteristic equation is:

$$
\tilde{W}(z)=\left(1-\bar{a}\left[z^{0}|\cdots| z^{-(k-1)}\right]^{T}\right)
$$

In robust sense: 


$$
\tilde{W}(z)=\left(1-\left[\bar{a}_{L}, \bar{a}_{U}\right]\left[z^{0}|\cdots| z^{-(k-1)}\right]^{T}\right)
$$

It is described in explicit form as:

$$
\tilde{W}(z)=\left(1-\left(\begin{array}{l}
{\left[\bar{a}_{0}, \bar{a}_{0}\right]+\left[\bar{a}_{1}, \bar{a}_{1}\right] z^{-1}+\cdots} \\
+\left[\bar{a}_{(k-1)}, \bar{a}_{(k-1)}\right] z^{-(k-1)}
\end{array}\right)\right)
$$

With the Kalman fuzzy filter, the different operation levels of the filter proposed must match inside the filter error criterion, with respect with the desired signal $y(k)$, the membership parameter selection of $\hat{\bar{a}}(k) \in \mathfrak{R}^{([1 \times 6], k)}$ into the filter structure, and the filter output $\hat{y}(k) \in \mathfrak{R}$. In accordance with a mathematical selection process into the fuzzy filter. The Figure $\mathbf{3}$ shows the desired signal $y(k) \in \mathfrak{R}$ approximation with the fuzzy filter output described as $\hat{y}(k) \in \mathfrak{R}$.

In accordance with the desired signal levels, the fuzzy stage makes a selection process by the fuzzy rules to get dynamically the parameter value (membership value) changing its values through time. Figure 4 shows this process schematically, in where the fuzzy stage that makes a classification process describing the parameter values into levels and dynamically selects the best correspondence with the reference process changes having the best signal approximation considering the lowest value error. In accordance to (9) and having five delays:

$$
\begin{gathered}
{\left[\underline{a_{0}}, \overline{a_{0}}\right]=[0.012,0.0125],\left[\underline{a_{1}}, \overline{a_{1}}\right]=[0.32,0.321],} \\
{\left[\underline{a_{2}}, \overline{a_{2}}\right]=[0.249,0.25],\left[\underline{a_{3}}, \overline{a_{3}}\right]=[0.357,0.36],} \\
{\left[\underline{a_{4}}, \overline{a_{4}}\right]=[0.24,0.241],\left[\underline{a_{5}}, \overline{a_{5}}\right]=[0.028,0.029],}
\end{gathered}
$$

has the form:

$$
\tilde{\mathrm{W}}(z)=1-\left(\begin{array}{l}
{[0.012,0.0125]+[0.32,0.321] \mathrm{z}^{-1}} \\
+[0.249,0.25] \mathrm{z}^{-2}+[0.357,0.36] z^{-3} \\
+[0.24,0.241] z^{-4}+[0.028,0.029] z^{-5}
\end{array}\right)
$$

The Kharitonov's polynomials considering Equation (10) with the fuzzy estimation regions:

$$
\begin{aligned}
& \tilde{W}(z)_{1}=0.18+0.09 z^{-2}+0.1 z^{-4}, \\
& \tilde{W}(z)_{2}=0.22+0.03 z^{-2}+0.16 z^{-4}, \\
& \tilde{W}(z)_{3}=0.32 z+0.3 z^{-3}+0.028 z^{-5}, \\
& \tilde{W}(z)_{4}=0.37 z^{-1}+0.23 z^{-3}+0.032 z^{-5}
\end{aligned}
$$

An observing in (19) that the polynomials coefficients do not have sign changes using the Ruth-Hurwitz criterion.

The Figure 5 has the state identification process

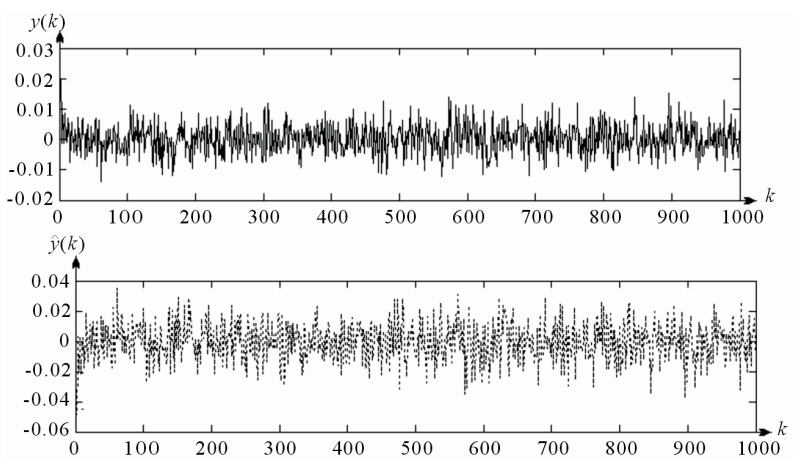

Figure 3. Desired signal and its approximation.

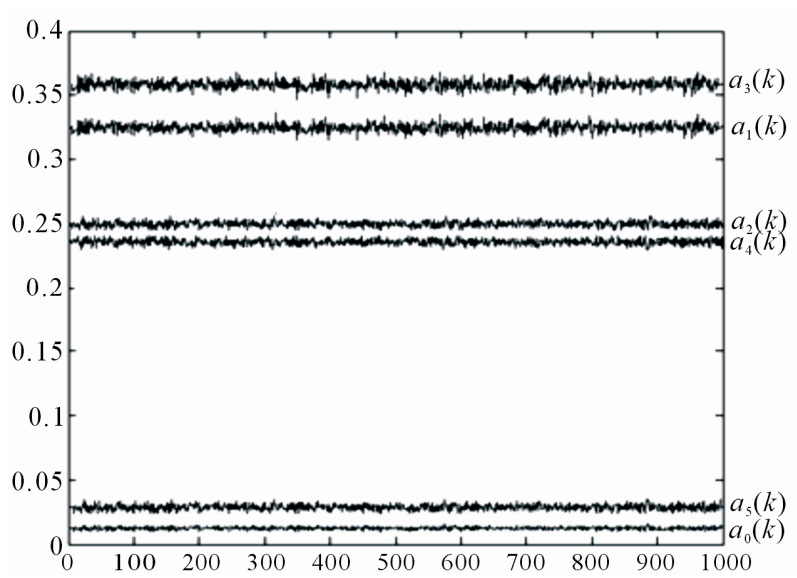

Figure 4. Membership value selection.
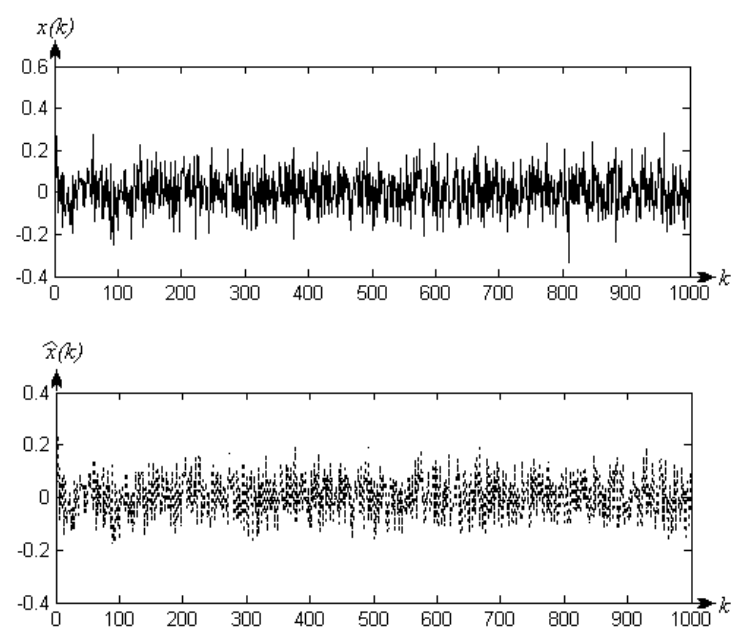

Figure 5. Internal identification states.

$x(k)$ of the reference model, using the fuzzy filter having a description of the internal process by $\hat{x}(k)$. This internal estate describes where the reference model information is, with respect with the dynamical changes.

With the fuzzy inference using the membership levels into the set of fuzzy rules the Figure 6 shows the membership classification in order to select the best answer 


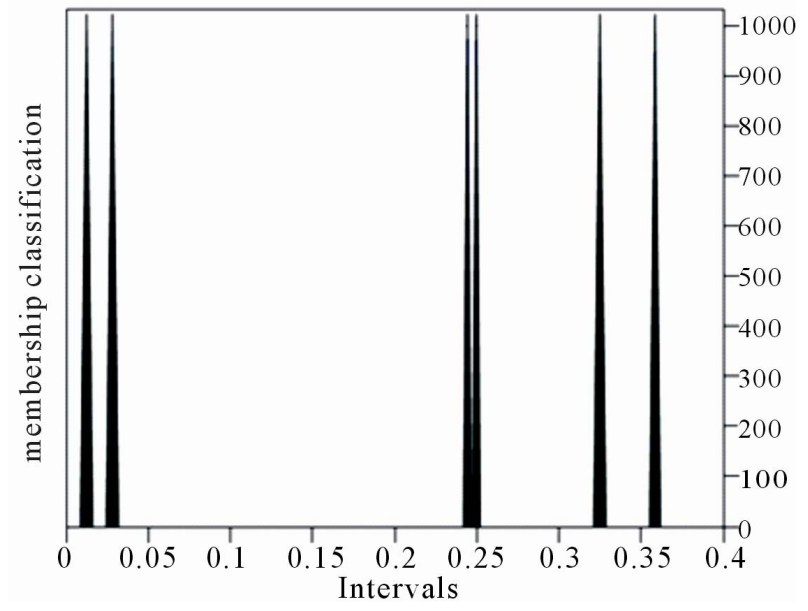

Figure 6. Error levels classification.

and have a the minimum error convergence.

Finally, in accordance with the fuzzy filter selection process the Figure 7 shows the different operational levels of the filter output described by $\hat{y}(k)$.The FDF describes a real signal into operational levels minimizing the error difference into the filter mechanism, as we can see the graphics above and into the Figure 8 that describe the filter response functional error in accordance with the desired signal using the error criterion.

The FDF can classify a signal, having an inference that interpret the input (desired signal) and get the best parameter value to approximate to the changes having the minimum error into the convergence.

\section{Conclusions}

The fuzzy filter type III having a signal characterization that adapts best to the external changes. In artificial intelligent systems, operations can be graded by the fuzzy stage using inference to interpret the input from an external process, and obtain the best membership value selected dynamically from the knowledge base, updating the filter parameter weights to get the best transition of the system applying into Kalman structure [16].

The Kharitonov's theory helps establish the analysis of the fuzzy filter process considering the maximum and minimum limits of the system to describe the stability of all possible combinations of the polynomials. Then with the Routh-Hurwitz criteria we can obtain the stability of each polynomial.

This work showed a simulation description of the FDF type III operation using the Matlab and the Kalman filter structure to integrate the fuzzy mechanism, considering three levels, having an accurate filtering time response with respect to the reference model.

The filter can be applied to, for example devices that interacts with our brains describing our external and in-

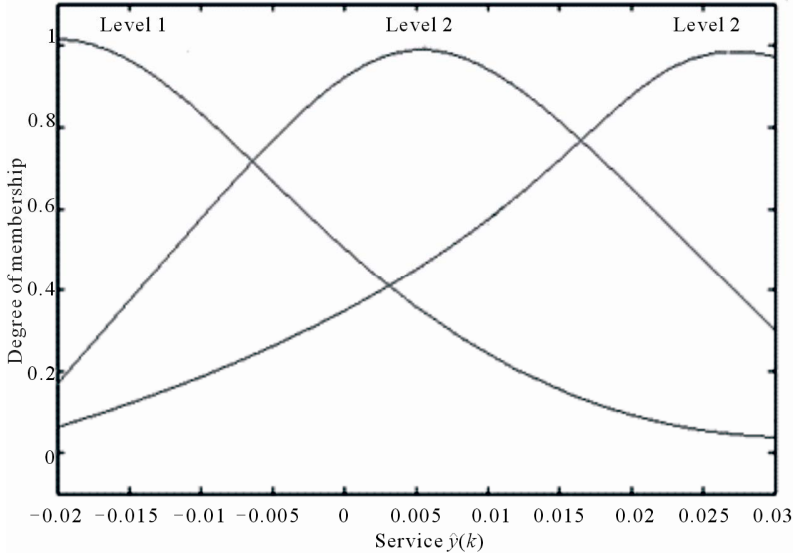

Figure 7. Filter service level.

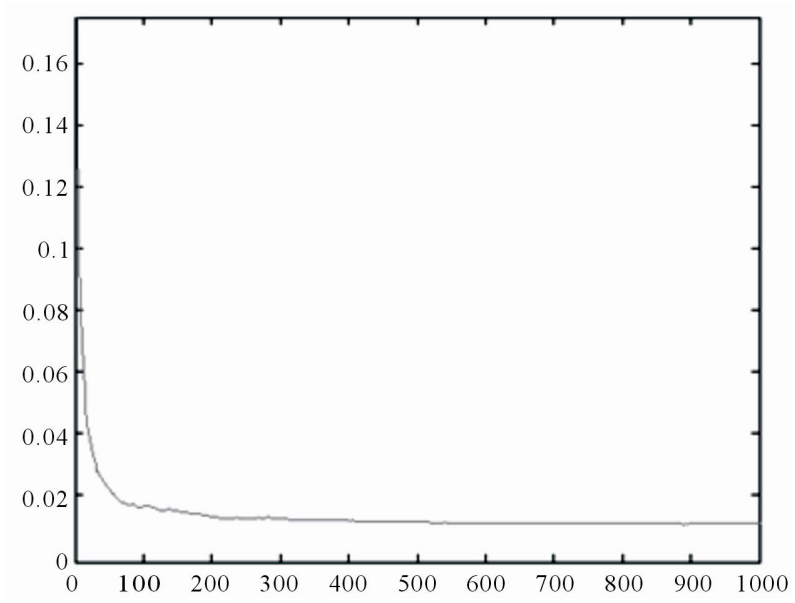

Figure 8. Fuzzy Filter Functional Error.

ternal processes, communicates with neurons to deduce the best action, as human biological processes and its external environment changes.

\section{REFERENCES}

[1] A. H. Sayed, "Fundamentals of Adaptive Filters," WileyIEEE, Hoboken, 2003.

[2] J. Smith and A. Eiben, "Introduction to Evolutionary Computing,” Springer, Dordrecht, 2003.

[3] T. Amble, "Logic Programming and Knowledge Engineering,” Addison Wesley, Boston, 1987.

[4] J. J. Medel, J. C. García and J. C. Sánchez, "Real-time Fuzzy Digital Filters (RTFDF) Properties for SISO Systems," Automatic Control and Computer Sciences, Vol. 41, No. 1, 2008, pp. 26-34.

[5] F. Yamakawa, "Fuzzy Neurons and Fuzzy Neural Networks,” 1989.

[6] D. Marcek, "Stock Price Forecasting: Statistical, Classical and Fuzzy Neural Networks,” Modeling Decisions for Artificial Intelligence, Springer Verlag, Berlin, 2004, pp. 
41-48.

[7] V. Kharitonov, "Robust stability analysis of time delay systems: A survey,” Annual Reviews in Control, Vol. 23, 1999, pp 185-196. doi.10.1016/S1367-5788(99)90087-1

[8] L. Zadeh, "Fuzzy Sets," Information and Control, Vol. 8, No. 3, 1965, pp. 338-353. doi:10.1016/S0019-9958(65)90241-XU

[9] C. L. Chen, G. Feng and X. P. Guan, "Delay Dependent Stability Analysis and Controller Synthesis for Discrete Time TS Fuzzy Systems with Time Delays," IEEE Transactions on Fuzzy Systems, Vol. 13, No. 5, 2005, pp. 630-643. doi:10.1109/TFUZZ.2005.856562U

[10] E. Onieva,V. Milanés, J. Pérez and T. Pedro, "Estimación de un Control Lateral Difuso de Vehículos,” RIAII, Vol. 7, No. 2, 2010, pp. 91-98. doi:10.4995/RIAI.2010.02.09U

[11] C. W. Tao and J. S. Taur, "Robust Fuzzy Control for a Plant with Fuzzy Lineal Model,” IEEE Transactions on Fuzzy Systems, Vol. 13, No. 1, 2005, pp. 30-41. doi:10.1109/TFUZZ.2004.839653U

[12] E. Mamdani, "Applications of Fuzzy Algorithms for Control of Simple Dynamic Plant," Proceedings of the IEEE, Vol. 121, No. 12, 1974, pp. 1585-1588.

[13] F. Gustafsson, "Adaptive Filtering and Change Detection,” John Wiley and Sons Ltd, Hoboken, 2000.

[14] K. M. Passino, "Fuzzy Control,” Addison Wesley, Boston, 1998.

[15] Ash, "Real Analysis and Probability," Academic Press
USA, 1970.

[16] T. Takagiand M. Sugeno, "Fuzzy Identification of Systems and Its Applications to Modelling and Control," IEEE Transactions on Systems, Man, and Cybernetics, Vol. 15, No. 1, 1986, pp. 116-132.

[17] L. Zadeh, "Maximizing Sets and Fuzzy Markoff Algorithms," IEEE Transactions on Systems, Man, and Cybernetics-Part C: Applications and Reviews, Vol. 28, No. 1, 1998, pp. 9-15. doi:10.1109/5326.661086U

[18] J. García,J. Medel andL. Guevara, "Filtrado Difuso en Tiempo Real,” Computación y Sistemas, Vol. 11, No. 4, 2008, pp. 390-401.

[19] J. García, J. Medel and J. Sánchez, "Evolutive Neural Net Fuzzy Filtering: Basic Description,” Journal of Intelligent Learning Systems and Applications, Vol. 2, No. 1, 2010, pp. 12-18.

[20] S. Haykin, “Adaptive Filtering,” Prentice Hall, UpperSaddle River, 2001.

[21] M. Margaliot and G. Langholz, "New Approaches to Fuzzy Modeling and Control Design and Analysis,” World Scientific, 2000. doi:10.1142/9789812792716U

[22] B. Rajen and M. Gopal, "Neuro-Fuzzy Decision Trees," International Journal of Neural Filters, Vol. 16, No. 1, 2006, pp. 63-68. doi:10.1142/S0129065706000470U

[23] M. Shannon, "A Mathematical Theory of Communication,” Bell Systems Technical Journal, Vol. 27, 1948, pp. 379-423\&623-656. 